\title{
Fusion of Hyperspectral and LiDAR Data Using Discriminant Correlation Analysis for Land Cover Classification
}

\author{
Farah Jahan, Jun Zhou, Senior Member, IEEE, Mohammad Awrangjeb, Senior Member, IEEE, Yongsheng Gao, \\ Senior Member, IEEE
}

\begin{abstract}
It is evident that using complementary features from different sensors is effective for land cover classification. Therefore, combining complementary information from Hyperspectral (HS) and Light Detection and Ranging (LiDAR) data can greatly assist in such applications. In this paper, we propose a model for land cover classification which extracts effective features representing different characteristics (e.g., spectral, geometrical/structural) of objects of interest from these two complementary data sources (e.g., HS and LiDAR) and fuse them effectively by incorporating dimensionality reduction technique. The HS bands are first grouped based on their joint entropy and structural similarity for group-wise spatial feature extraction. The spectral and spatial features from HS are then fused in parallel via discriminant correlation analysis (DCA) method for each band group. This is followed by a multisource fusion step between the spatial features extracted from HS and LiDAR data using DCA. The resultant features from both band-group fusion and multisource fusion steps are concatenated with several other features extracted from HS and LiDAR data. In the proposed model, DCA fusion produces discriminative features by eliminating between-class correlations and confining withinclass correlations. We compare the performance of our feature extraction and fusion scheme using random forest and support vector machine classifiers. We also compare our approach with several state-of-the-art approaches on two benchmark land cover datasets and show that our approach outperforms the alternatives by a large margin.
\end{abstract}

Index Terms-Hyperspectral, LiDAR, fusion, DCA, Multisource.

\section{INTRODUCTION}

Accurate land cover classification is a primary step for many applications such as forest inventory management [1], biomass estimation [2], urban planning [3], and global environment change [4]. Remote sensing technology has been widely used for land cover classification [3, 5]. It facilitates rapid and accurate data acquisition with a small amount of required resources compared to the ground survey [4].

Land cover classification can be based on the optical data only $[6,7]$, or on a combination of multimodal remote sensing data captured by both passive multispectral and hyperspectral (HS) sensors and active LiDAR and synthetic aperture radar (SAR) systems [1, 2, 8]. Several studies show that integration of multisource or multimodal data can improve the performance of land cover classification [3, 9]. Among

F. Jahan, J. Zhou, M. Awrangjeb and Y. Gao are with the Institute of Integrated and Intelligent Systems, Griffith University, Nathan, Australia. Corresponding author: Farah Jahan (farah.jahan@griffithuni.edu.au). various sensors, HS sensors capture hundreds of narrow bands from visible to short-wave infrared wavelengths and provide contiguous spectral information of ground objects. On the other hand, LiDAR is known as a vital method for characterising vertical structures, including height and volume. It sends laser signals to targets and measures their height as well as the intensity of the reflected pulses which depend on the nature of the reflecting material. HS and LiDAR data have been integrated in several studies so that their complementary information can be utilised to improve forest analysis and land cover classification [3, 4, 9-11].

Feature extraction is one of the key steps for HS and LiDAR data fusion. Bigdeli et al. [12] extracted features separately from HS and LiDAR data and applied a particle swarm optimisation method to select features. Then a fuzzy multi-classifier system based on decision template was used to classify HS and LiDAR features. Several recent works explored the capability of spatial information for land cover classification. Ghamisi et al. [11] used attribute profile (AP) and Khodadadzadeh et al. [13] used morphological attribute profiles (MAPs) to extract spatial information from $\mathrm{HS}$ and LiDAR data. The markov random field (MRF) was used to promote spatial smoothness in classification. Ghamisi et al. [14] used kernel PCA for extracting spectral feature from HS and extinction profile (EP) for extracting spatial features from both HS and LiDAR data.

In most cases, the fusion is undertaken at pixel-level. This can be done either by directly stacking the features extracted from both the HS and LiDAR data [3, 4, 11-13, 15] or by extracting features first and then adopting an effective fusion model [14, 16]. In the first case, high dimensional data is generated by fusion leading to curse of dimensionality and overfitting classification models. Therefore, a key step and challenge here is to reduce such high dimensional fused data. Liao et al. [17] proposed a generalised graph-based feature fusion method which fused spectral information from HS data with morphological profiles of HS and LiDAR data. The fusion method also incorporated dimensionality reduction. Ghamisi et al. [14] used a graph based feature fusion to reduce the high dimensionality of feature vector. The fused features form the input to convolutional neural network (CNN) and Random Forest (RF) classifiers. Rasti et al. [16] proposed a sparse and low-rank technique to reduce the spectral redundancy when fusing features from HS and LiDAR data. Though feature extraction and fusion approaches showed good performance 


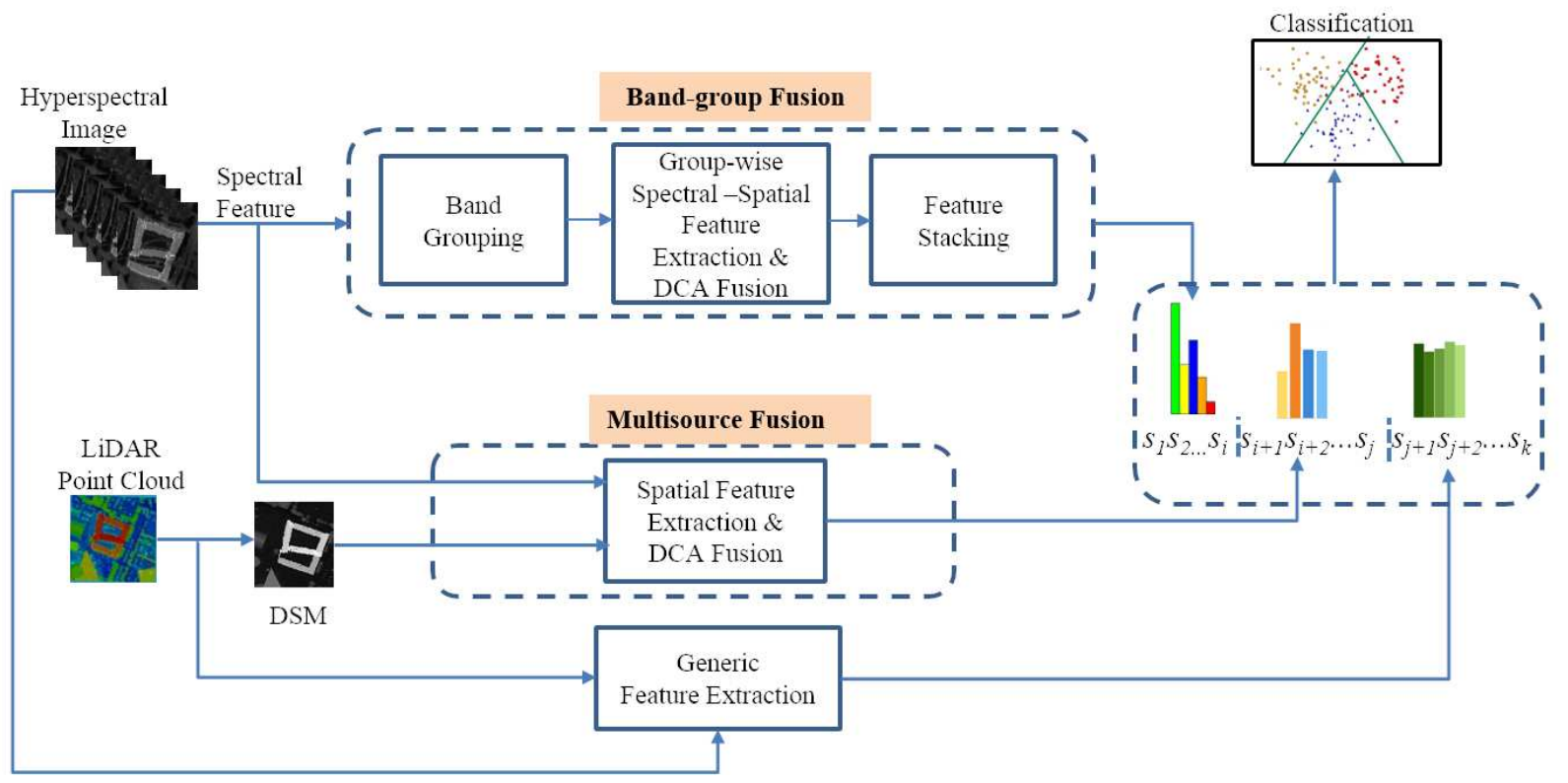

Fig. 1. The architecture of the proposed method.

in land cover classification, an effective exploration of both spectral and spatial information in multimodal data is still a challenging task.

Being motivated by the success of correlation and discriminant analysis based feature fusion [18, 19], in this paper, we propose a novel feature extraction and fusion method where DCA plays the role of basic fusion unit. This method incorporates three key tasks e.g., band-group fusion, multisource fusion, and generic feature extraction. Band-group fusion reduces redundant information in high dimensional HS data and extracts discriminative feature from the band group. We group HS bands based on their joint entropy and structural similarity and apply principal component analysis (PCA) on each group to reduce the number of bands. Then differential attribute profile (DAP) [20] is extracted from each group to generate spatial features. Both spatial and spectral features from individual groups are fused using DCA. The results from individual groups are concatenated to generate a feature vector for the HS data. Multisource fusion is employed to fuse spatial feature from both HS and LiDAR data using DCA. We also extract several generic pixel-wise features from the HS and LiDAR data which are arranged sequentially to form an additional feature vector. Finally, we concatenate the features generated by band-group fusion, multisource fusion and generic feature extraction steps to get a final signature. A summary of our method is given in Fig. 1.

The main contributions of this paper are three-fold:

- We propose a novel framework that combines features generated by band-group fusion, multisource fusion and generic feature extraction. Each step is designed to target different properties of the multimodal data.

- We introduce DCA fusion for combining data from two different but correlated feature spaces. This fusion method maximises the pairwise correlations between two feature sets. It also removes the between-class correla- tions and confines the correlations within the classes [18].

- We explore the distinctive contribution from band groups of HS data in band-group fusion. The band grouping method groups HS bands based on their joint entropy and structural similarity. Spatial features are extracted from each group and fused with their spectral features which results in discriminative and low dimensional features from HS data.

- We undertake comprehensive experiments to validate the effectiveness of our approach and the contribution from each key step. We also compare our method with several state-of-the-art approaches.

The rest of the paper is organised as follows. Section II provides a detailed description of the proposed method. The experimental results on two real HS and LiDAR datasets are presented and discussed in Section III. Finally, Section IV concludes this paper with a few observations.

\section{Methodology}

In this section, we describe the details of the proposed HS and LiDAR data fusion approach. As shown in Fig. 1, our method has three key tasks: band-group fusion, multisource fusion, and generic feature extraction.

\section{A. Band-group Fusion}

We employ a band-group fusion approach to generate discriminative features from the HS cube, a stack of bands. This task consists of four steps: band grouping, band reduction, spatial feature extraction and DCA fusion. As shown in Fig. 2(a), the DCA fusion step is applied to each band group separately.

Here, the target of processing is an HS cube $\mathcal{H} \in \mathbb{R}^{M \times N \times R}$, where $M$ and $N$ are height and width of spatial dimensions respectively, and $R$ is the number of bands. We first generate 


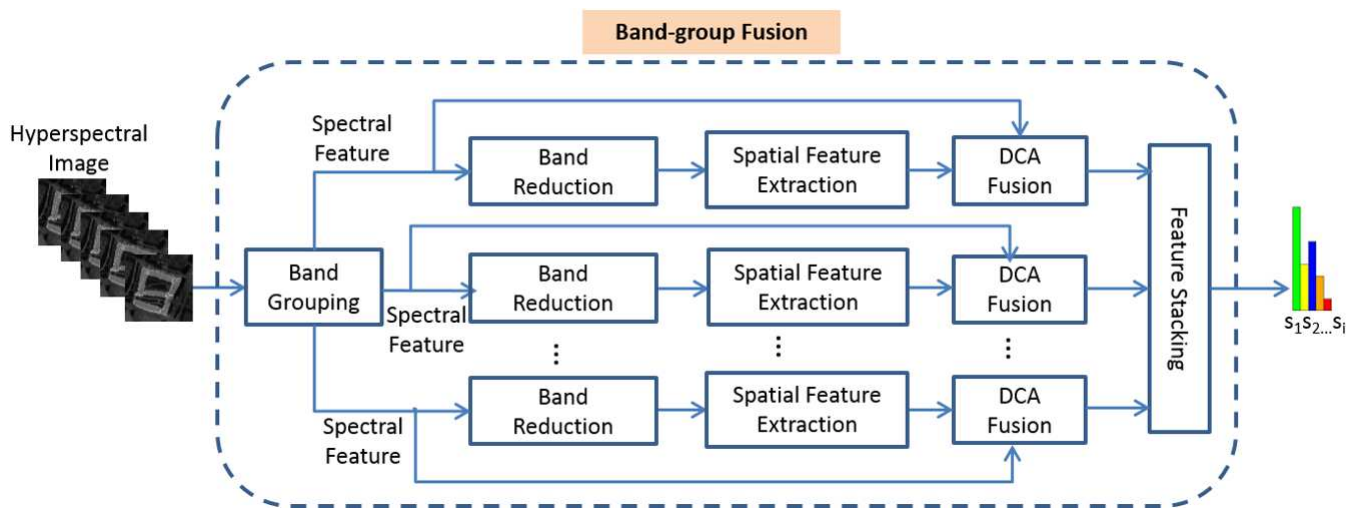

(a)

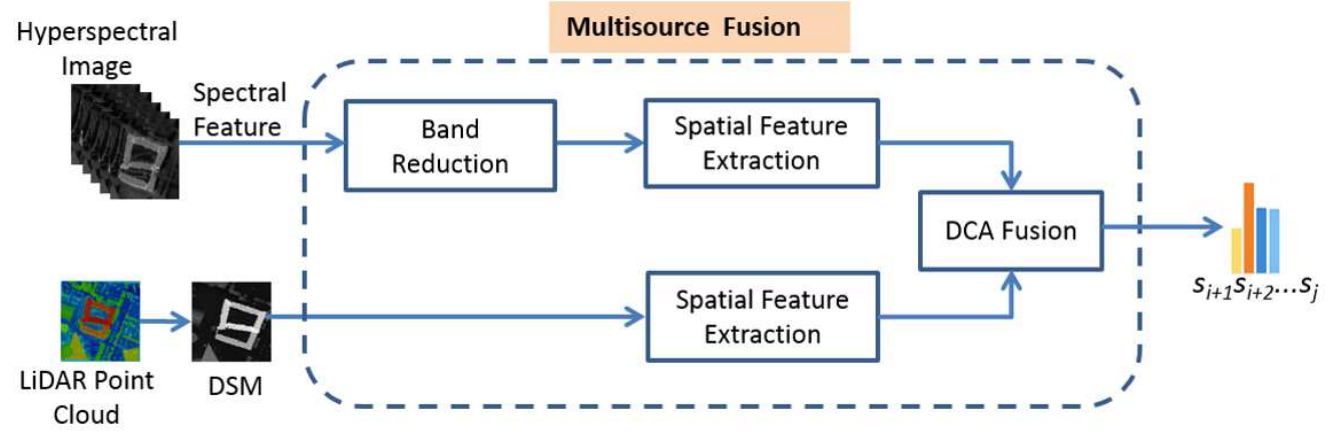

(b)

Fig. 2. Steps for: (a) Band-group fusion and (b) Multisource fusion

$T$ band groups from $\mathcal{H}$, where the $t$-th $(t \in T)$ band group has $b_{t}$ and $s_{t}$ features from the spectral and spatial domains respectively or from two data modalities. For the $t$-th band group, the feature vector of a pixel with coordinate $(x, y)$ is obtained from the fusion operation. The fusion operation can be expressed by

$$
\mathbf{x}_{t}=\mathcal{F}\left(\alpha^{1}, \cdots, \alpha^{b_{t}}, \gamma^{1}, \cdots, \gamma^{s_{t}}\right),
$$

where $\mathcal{F}$ is a fusion operator. $\alpha^{1}, \cdots, \alpha^{b_{t}}$ and $\gamma^{1}, \cdots, \gamma^{s_{t}}$ are two different sets of features. The definition can be expanded to parallel band-group fusion setting, where $R$ bands are distributed into multiple groups and features from each group are aimed to be fused simultaneously as

$$
\begin{gathered}
\mathbf{x}_{1}=\mathcal{F}\left(\alpha^{1}, \cdots, \alpha^{b_{1}}, \gamma^{1}, \cdots, \gamma^{s_{1}}\right) \\
\vdots \\
\mathbf{x}_{t}=\mathcal{F}\left(\alpha^{1}, \cdots, \alpha^{b_{t}}, \gamma^{1}, \cdots, \gamma^{s_{t}}\right) \\
\vdots \\
\mathbf{x}_{T}=\mathcal{F}\left(\alpha^{1}, \cdots, \alpha^{b_{T}}, \gamma^{1}, \cdots, \gamma^{s_{T}}\right) .
\end{gathered}
$$

The fusion method is explained in detail in Section II-A3.

1) Band Grouping: We cluster the bands in an HS cube into several groups based on their similarity. To this end, we use joint entropy and structural similarity index [21] among the bands.

The joint entropy between two bands measures their redundancy. To estimate the joint entropy, we first calculate the gradient magnitude at each pixel for every band. Let, the gradient magnitude of a pixel in band $r$ located at $(x, y)$ be expressed by $\mathbf{G}_{x, y}^{r}$. Then, we calculate the mean gradient at a pixel for $R$ bands as

$$
\overline{\mathbf{G}_{x, y}}=\frac{\sum_{r=1}^{R} \mathbf{G}_{x, y}^{r}}{R}
$$

where $x=1 \ldots M, y=1 \ldots N$. The joint entropy $\beta_{r}$ for the $r$-th band is then defined by

$$
\beta_{r}=-\sum_{y=1}^{N} \sum_{x=1}^{M} p\left(\mathbf{G}_{x, y}^{r}, \overline{\mathbf{G}_{x, y}}\right) \log p\left(\mathbf{G}_{x, y}^{r}, \overline{\mathbf{G}_{x, y}}\right),
$$

where $p($.$) denotes the probability density function of the given$ random variables.

Structural similarity index [21] measures the similarity between two images. In our approach, we calculate the similarity between each band and the spectral-mean of the whole HS cube. The spectral-mean of the cube is a two-dimensional image calculated as

$$
\overline{\mathbf{H}}=\frac{\sum_{r=1}^{R} \mathbf{H}^{r}}{R}
$$

where $\mathbf{H}^{r}$ and $\overline{\mathbf{H}}$ represent $r$-th band and mean of the cube respectively. $\mathbf{H}^{r}$ and $\overline{\mathbf{H}}$ both are two-dimensional images. We 
calculate mean, standard deviation and cross-variance of $\mathbf{H}^{r}$ and $\overline{\mathbf{H}}$ using Equations (6), (7) and (8) respectively.

$$
\begin{gathered}
\mu_{\mathbf{H}^{r}}=\frac{1}{M N} \sum_{x=1}^{M} \sum_{y=1}^{N} \mathbf{H}_{x, y}^{r} \\
\mu_{\overline{\mathbf{H}}}=\frac{1}{M N} \sum_{x=1}^{M} \sum_{y=1}^{N} \overline{\mathbf{H}}_{x, y} \\
\sigma_{\mathbf{H}^{r}}=\left(\frac{1}{M N-1} \sum_{x=1}^{M} \sum_{y=1}^{N}\left(\mathbf{H}_{x, y}^{r}-\mu_{\mathbf{H}^{r}}\right)^{2}\right)^{\frac{1}{2}} \\
\sigma_{\overline{\mathbf{H}}}=\left(\frac{1}{M N-1} \sum_{x=1}^{M} \sum_{y=1}^{N}\left(\overline{\mathbf{H}}_{x, y}-\mu_{\overline{\mathbf{H}}}\right)^{2}\right)^{\frac{1}{2}},
\end{gathered}
$$

and

$$
\sigma_{\mathbf{H}^{r} \overline{\mathbf{H}}}=\left(\frac{1}{M N-1} \sum_{x=1}^{M} \sum_{y=1}^{N}\left(\mathbf{H}_{x, y}^{r}-\mu_{\mathbf{H}^{r}}\right)\left(\overline{\mathbf{H}}_{x, y}-\mu_{\overline{\mathbf{H}}}\right)\right)^{\frac{1}{2}},
$$

where $\mu_{\mathbf{H}^{r}}$ and $\mu_{\overline{\mathbf{H}}}$ are the means of $\mathbf{H}^{r}$ and $\overline{\mathbf{H}}$ respectively, $\sigma_{\mathbf{H}^{r}}$ and $\sigma_{\overline{\mathbf{H}}}$ are the standard deviations of $\mathbf{H}^{r}$ and $\overline{\mathbf{H}}$ respectively, and $\sigma_{\mathbf{H}^{r} \overline{\mathbf{H}}}$ is the cross-variance between $\mathbf{H}^{r}$ and $\overline{\mathbf{H}}$.

For the similarity index, we consider luminance variation $l\left(\mathbf{H}^{r}, \overline{\mathbf{H}}\right)$, contrast variation $c\left(\mathbf{H}^{r}, \overline{\mathbf{H}}\right)$ and structural variation $s\left(\mathbf{H}^{r}, \overline{\mathbf{H}}\right)$ as given in Equations (9), (10) and (11) respectively.

$$
\begin{array}{r}
l\left(\mathbf{H}^{r}, \overline{\mathbf{H}}\right)=\frac{2 \mu_{\mathbf{H}^{r}} \mu_{\overline{\mathbf{H}}}+C_{1}}{\mu_{\mathbf{H}^{r}}^{2}+\mu_{\overline{\mathbf{H}}}^{2}+C_{1}}, \\
c\left(\mathbf{H}^{r}, \overline{\mathbf{H}}\right)=\frac{2 \sigma_{\mathbf{H}^{r}} \sigma_{\overline{\mathbf{H}}}+C_{2}}{\sigma_{\mathbf{H}^{r}}^{2}+\sigma_{\overline{\mathbf{H}}}^{2}+C_{2}},
\end{array}
$$

and

$$
s\left(\mathbf{H}^{r}, \overline{\mathbf{H}}\right)=\frac{\sigma_{\mathbf{H}^{r} \overline{\mathbf{H}}}+C_{3}}{\sigma_{\mathbf{H}^{r}} \sigma_{\overline{\mathbf{H}}}+C_{3}} .
$$

$C_{1}, C_{2}$ and $C_{3}$ used in the above Equations 9 to 11 are constants. Their values are set as $C_{1}=(0.01 * L)^{2}, C_{2}=$ $(0.03 * L)^{2}$ and $C_{3}=C_{2} / 2$, where $L$ is a constant which is represented by the dynamic range of the input image [21]. Finally, we calculate the structural similarity metric $\lambda\left(\mathbf{H}^{r}, \overline{\mathbf{H}}\right)$ which is the combination of Equations (9)-(11) and can be expressed by

$$
\lambda\left(\mathbf{H}^{r}, \overline{\mathbf{H}}\right)=\frac{\left(2 \mu_{\mathbf{H}^{r}} \mu_{\overline{\mathbf{H}}}+C_{1}\right)\left(2 \sigma_{\mathbf{H}^{r} \overline{\mathbf{H}}}+C_{2}\right)}{\left(\mu_{\mathbf{H}^{r}}^{2}+\mu_{\overline{\mathbf{H}}}^{2}+C_{1}\right)\left(\sigma_{\mathbf{H}^{r}}^{2}+\sigma_{\overline{\mathbf{H}}}^{2}+C_{2}\right)} .
$$

The joint entropy and the structural similarity index are used together to measure the similarity between two images. In order to find distance metric between them, we use the inverse of joint entropy and structural similarity index individually. So, a band in the Euclidean space can be represented using a point $\left(\beta^{-1}, \lambda^{-1}\right)$. Then, we apply Expectation-Maximization (EM) Clustering using Gaussian Mixture Models (GMM) to group them into clusters.
2) Band Reduction and Feature Extraction: The band grouping leads to a group of correlated bands, so each group contains a large amount of redundant information. The dimensionality and correlations of each band group need to be reduced so as to enable faster computation and feature extraction. We apply principal component analysis [22] on every band group to reduce their spectral dimension.

We are interested in spatial structures in an image because spatial structures are perceptually significant for land cover classification. Morphological connected filters can be used to extract spatial features which efficiently retrieve structures without any distortion [20]. In this study, we exploit DAP [20] to achieve this goal.

Since, no single filter size is suitable to handle the structures of all the objects in an image, morphological profiles are generated by a sequence of opening and closing with structuring element (SE) of increasing size to cope with scale problem. Given an image $I$, let us consider "area" as an attribute having $h$ different attribute values. The attribute profile for this attribute is calculated by applying opening and closing operations on the image for each of the $h$ attribute values. The resulting attribute profile is a stack of $2 h+1$ planes $(h$ planes from the closing profile, the original image plane, and $h$ planes from the opening profile.) DAP [20] stores the residuals of the subsequent increasing transformations applied to the image. Since, important components of the profiles are more evident in DAP, it is more practical to use DAP for obtaining important information.

Given $\rho$ significant principal components produced by the $t$ th band group. For each plane, DAP generates $2 h+1$ profiles, i.e., $n_{t}=\rho \times(2 h+1)$ profiles or planes for the band group. In our approach, we use three attribute values as area and three attribute values as diagonal length of bounding boxes; thus produce 13 profiles.

3) Discriminant Correlation Analysis: After band grouping, we extract DAP [20] from the reduced bands of each band group and fuse it with the raw spectral features of the same band group. To complete this fusion task, we introduce DCA which incorporates class separation knowledge by combining correlation and discriminant analysis. Here, we aim to find the maximum separation between classes and minimum separation within classes. So, the task is completed in two steps: increasing class separation and feature combination. The DCA fusion is not only used in a band-group fusion, but also in the multisource fusion to fuse spatial features from HS and LiDAR data.

Let us suppose, we are given two feature sets $\mathbf{F}$ and $\mathbf{K}$ extracted from $n$ samples (pixels) which are grouped into $C$ different classes. Suppose, the $t^{t h}$ sample in $\mathbf{F}$ and $\mathbf{K}$ is represented by $\alpha_{t}^{1}, \ldots, \alpha_{t}^{b_{t}}$ and $\gamma_{t}^{1}, \ldots, \gamma_{t}^{s_{t}}$ respectively, where $b_{t}$ and $s_{t}$ are the length of the vectors. The centroid of class $c$ is calculated for the first feature set $\mathbf{F}$ as

$$
\bar{\alpha}_{c}=\frac{1}{n_{c}} \sum_{i=1}^{n_{c}} \alpha_{c}^{i}
$$

where $n_{c}$ is the number of samples or feature vectors in class $c$ and $\alpha_{c}^{i}$ is the $i$-th feature vector in the class. So, the between- 
class scatter matrix [23] for the feature set $\mathbf{F}$ of $C$ classes can be calculated as

$$
\mathbf{B}_{\alpha}=\sum_{c=1}^{C} n_{c}\left(\bar{\alpha}_{c}-\bar{\alpha}\right)\left(\bar{\alpha}_{c}-\bar{\alpha}\right)^{T},
$$

where $\bar{\alpha}$ is the mean feature vector of the feature set $\mathbf{F}$.

If the classes are well-separated and $\mathbf{U}$ is the matrix of orthogonal eigenvectors of $\mathbf{B}_{\alpha}$ and $\mathbf{D}$ is the matrix of real and non-negative eigenvalues sorted in decreasing order, we can get:

$$
\mathbf{U}^{T} \mathbf{B}_{\alpha} \mathbf{U}=\mathbf{D} \text {. }
$$

So, $\mathbf{W}_{\alpha}=\mathbf{U D}^{-1 / 2}$ is the transformation matrix that unitises $\mathbf{B}_{\alpha}$, that is:

$$
\begin{gathered}
\mathbf{W}_{\alpha}^{T} \mathbf{B}_{\alpha} \mathbf{W}_{\alpha}=\mathbf{I} \\
\mathbf{F}^{\prime}=\mathbf{W}_{\alpha}{ }^{T} \mathbf{F}
\end{gathered}
$$

where $\mathbf{F}^{\prime}$ is the projection of $\mathbf{F}$ in a space considering $f$ principal components corresponding to $f$ largest non-zero eigen values in $\mathbf{W}_{\alpha}$. A similar approach is applied to the second feature set $\mathbf{K}$ to transform it into $\mathbf{K}^{\prime}$ :

$$
\mathbf{K}^{\prime}=\mathbf{W}_{\gamma}^{T} \mathbf{K}
$$

where $\mathbf{W}_{\gamma}$ is the transformation matrix derived from between class scatter matrix of the second feature set $\mathbf{K}$. The betweenclass scatter matrix of $\mathbf{K}$ can be calculated as follows:

$$
\mathbf{B}_{\gamma}=\sum_{c=1}^{C} n_{c}\left(\bar{\gamma}_{c}-\bar{\gamma}\right)\left(\bar{\gamma}_{c}-\bar{\gamma}\right)^{T}
$$

where the notations have usual meaning from contemporary first set.

In our fusion step, $\mathbf{F}$ and $\mathbf{K}$ are either taken from the same source (band groups from the same image for band-group fusion) or extracted at the same location (data from different sensors for multisource fusion), these features are correlated. In such case, direct multiplication does not increase the discriminability of features. Therefore, we first concatenate, e.g., $\mathbf{E}=\left[\begin{array}{ll}\mathbf{F}^{\prime} & \mathbf{K}^{\prime}\end{array}\right]$.

At this stage, we aim to extract mutual information from these two sets. This can be achieved by performing singular value decomposition on the concatenated feature set. To increase the correlation between features in two building blocks of $\mathbf{E}$, we calculate covariance of $\mathbf{E}$ as $\mathbf{E}_{c o v}$ and then factorise the covariance matrix. The factorisation of $\mathbf{E}_{c o v}$ is performed using singular value decomposition as follows:

$$
\begin{gathered}
\mathbf{E}_{c o v}=\mathbf{P} \boldsymbol{\Sigma} \mathbf{Q}^{T} \\
\left(\mathbf{P} \Sigma^{\frac{-1}{2}}\right)^{T} \mathbf{E}_{c o v}\left(\mathbf{Q} \boldsymbol{\Sigma}^{\frac{-1}{2}}\right)=\mathbf{I},
\end{gathered}
$$

where $\boldsymbol{\Sigma}$ is a diagonal matrix, $\mathbf{I}$ is an identity matrix, $\mathbf{P}$ and $\mathbf{Q}$ are unitary and orthonormal matrices respectively. Thus, the transformation matrices for transforming $\mathbf{E}$ can be defined using the right singular matrix $\mathbf{W}=\mathbf{Q} \boldsymbol{\Sigma}^{-\frac{1}{2}}$. In this way, the final projection of $\mathbf{E}$ can be obtained as follows:

$$
\mathbf{X}=\mathbf{W} * \mathbf{E} .
$$

Suppose DCA fusion generates $\mathbf{X}^{t}$ for the $t$-th band group in the band-group fusion. We can concatenate the feature vectors from $T$ band groups to generate the final feature of HS data:

$$
\mathbf{S}=\mathbf{X}^{1}\left\|\mathbf{X}^{2}\right\| \ldots \| \mathbf{X}^{T}
$$

\section{B. Multisource Fusion}

In this task, our aim is to fuse HS and LiDAR data. We extract spatial information from the whole HS data which is expected to contain different scene structures. At first, a new hyperspace is created by applying PCA on HS cube and then the cube is projected onto the new space which gives one or more planes considering PCs having at least $99 \%$ of variances. Spatial attributes are then extracted from both the transformed HS plane(s) and LiDAR DSM using the DAP algorithm. The obtained spatial information from both sources is fused using DCA which has been described in band-group fusion section. Fig. 2(b) shows the steps of multisource fusion.

\section{Generic Feature Extraction (GFeat)}

We derive a total of eight generic features from the HS and LiDAR data [24]. Among them, Normalised Difference Vegetation Index (NDVI) [3, 25] and Entropy (E) are extracted from HS. NDVI evaluates whether the target being observed contains live green vegetation. Entropy $(E)$ characterises the randomness of the texture. Note that the calculation of entropy requires a grayscale image. Given an HS cube, we select three bands from the cube and generate a pseudo-color RGB image. Then the RGB image is transformed to a gray scale image using a luminosity conversion function [26]. The entropy is calculated at each pixel centered in a patch of $9 \times 9$ of the grayscale image from a local neighborhood [26].

Six features are extracted from LiDAR, including Digital Surface Model (DSM), Digital Elevation Model (DEM), Normalised Digital Surface Model (nDSM), difference between the first and last LiDAR pulse return (PD), intensity of the LiDAR return and entropy of nDSM. DSM captures the natural elevation of the earth surface with objects on it. DEM is the representation of the bare-earth terrain without any object. $\mathrm{nDSM}$ is the difference between bare earth and first LiDAR return reflected by an object on earth. PD is the difference between first and last LiDAR pulse return of a pixel. It gives important information related to the solidness of the surface, and can distinguish trees and buildings with the same height, for example. Intensity is the strength of the LiDAR return pulse. nDSM Entropy measures the entropy calculated from the $n D S M$ data and calculated at each pixel centered in a patch of $9 \times 9$ of the nDSM image from a local neighorhood. Finally, we concatenate these eight features to form a generic feature vector for classification purpose. We concatenate these eight features to form part of the final feature vector.

\section{EXPERIMENTS AND ANALYSIS}

In this section, we present the experimental results on two real-world datasets, and compare our method with several state-of-the-art approaches. 


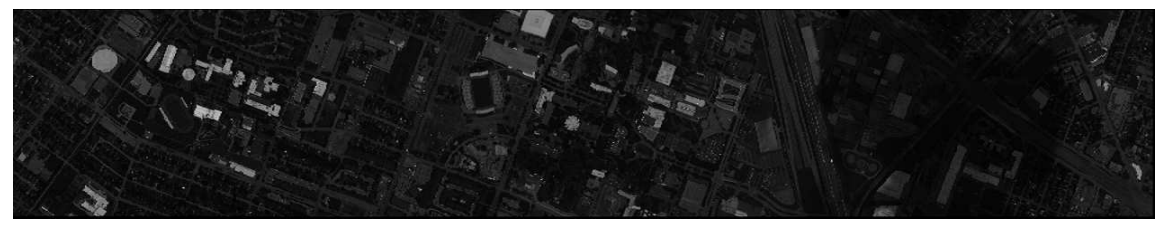

(a)

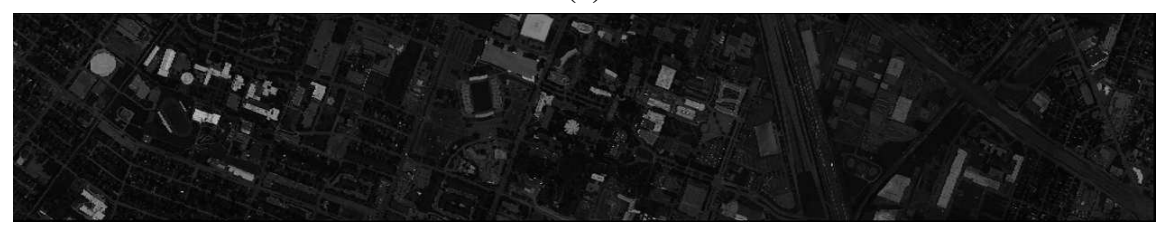

(b)

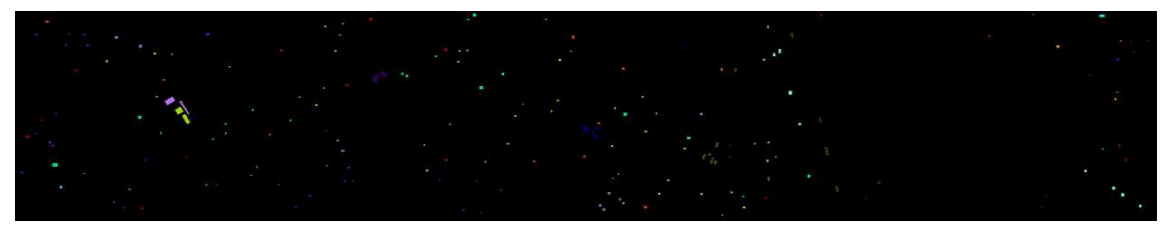

(c)

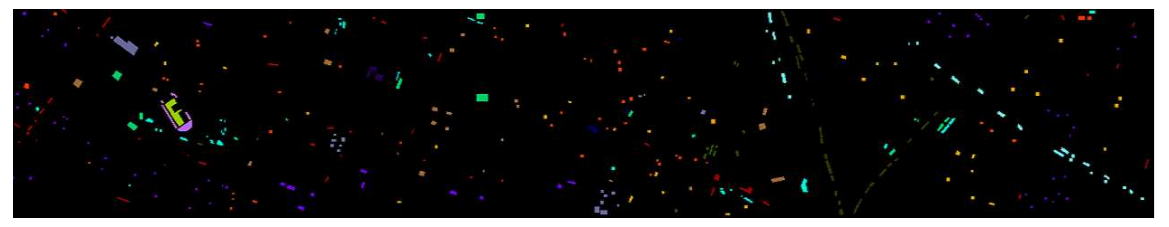

(d)

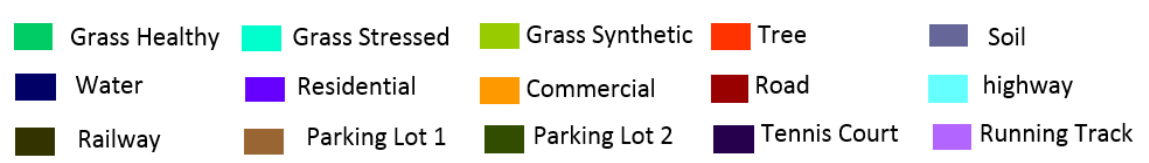

Fig. 3. Band 30 of the hyperspectral data of Houston. (a) Original image, (b) Shadow removed image (c) Training samples in (b), (d) Test samples in (b).

\section{A. Data Description}

1) Houston Data: The Houston dataset consists of a hyperspectral image and a LiDAR derived DSM. This dataset was distributed at the 2013 GRSS data fusion contest [27]. Both HS and LiDAR data were collected over the University of Houston campus and the neighbouring urban area. The HS data was acquired by a compact airborne spectrographic imager on June 23, 2012 and the average height of the sensor above ground was $5500 \mathrm{ft}$. The LiDAR data was acquired on June 22, 2012 and the average height of the sensor above ground was $2000 \mathrm{ft}$. The size of both HS and LiDAR data is $349 \times 1905$ pixels with the spatial resolution of $2.5 \mathrm{~m}$. The HS dataset consists of 144 spectral bands ranging from $0.38 \mu \mathrm{m}$ to $1.05 \mu \mathrm{m}$. The 15 classes of interests are Grass Healthy, Grass Stressed, Grass Synthetic, Tree, Soil, Water, Residential, Commercial, Road, Highway, Railway, Parking Lot 1, Parking Lot 2, Tennis Court, and Running Track. The distribution of training and testing samples of 15 different classes are shown in Table I. The "Parking Lot 1" class includes parking garages at the ground level and in elevated areas, while the "Parking Lot 2" class corresponds to parked vehicles.

Fig. 3 shows RGB composite image of Houston Data, location of training and testing samples. Fig. 3(a) is the RGB image obtained from the original HS data. Fig. 3(b) is obtained after shadow removal from Fig. 3(a) by Prof. Naoto Yokoya from Technical University of Munich. We use the image in Fig. 3(b) in our experiment for better visibility of object structure. To make the results fully comparable with other works in literature we use the standard training and testing set given with the Houston dataset.

Fig. 5 shows the representation of additional features derived from LiDAR point cloud and hyperspectral image of Houston Data.

2) GU Data: The second dataset is provided by the Spectral Imaging Lab at Griffith University. It consists of a hyperspectral image and corresponding LiDAR point cloud collected from Yarraman State Forest, Queensland, Australia and its adjacent area. The total area was around $8 \mathrm{~km}^{2}$. The hyperspectral data consists of 62 bands ranging from $408.54 \mathrm{~nm}$ to $990.62 \mathrm{~nm}$ wavelength, where the spectral resolution is $8.94 \mathrm{~nm}$ to $9.81 \mathrm{~nm}$. The spatial resolution of the dataset is $0.5 \mathrm{~m}$. For the LiDAR point cloud the number of return is 6 and average point spacing is $0.2 \mathrm{~m}$.

We collect training and testing samples from 33 different areas of the image. We crop hyperspectral data using the open source tool Barista, and process the LiDAR data using MARS 7 and ENVI 5.3 to generate DSM and DEM. The selected areas cover five classes of interest e.g., "Road", "Tree", "Grass", "Water" and "Soil". Out of the 33 areas, we arbitrarily select the points from 5 areas for training and remaining 28 areas 
TABLE I

HOUSTON DATASET: NUMBER OF TRAINING AND TESTING SAMPLES PER CLASS.

\begin{tabular}{|c|c|c|}
\hline & \multicolumn{2}{|c|}{ Number of Samples } \\
\hline Class Name & Training & Testing \\
\hline Grass healthy & 198 & 1053 \\
\hline Grass stressed & 190 & 1064 \\
\hline Synthetic grass & 192 & 505 \\
\hline Trees & 188 & 1056 \\
\hline Soil & 186 & 1056 \\
\hline Water & 182 & 143 \\
\hline Residential & 196 & 1072 \\
\hline Commercial & 191 & 1053 \\
\hline Road & 193 & 1059 \\
\hline Highway & 191 & 1036 \\
\hline Railway & 181 & 1054 \\
\hline Parking lot1 & 192 & 1041 \\
\hline Parking lot 2 & 184 & 285 \\
\hline Tennis court & 181 & 247 \\
\hline Running track & 187 & 473 \\
\hline Total & 2832 & 12197 \\
\hline \multicolumn{2}{|c}{}
\end{tabular}

TABLE II

GU DATASET: DISTRIBUTION OF TRAINING AND TESTING SAMPLES.

\begin{tabular}{|c|c|c|}
\hline & \multicolumn{2}{|c|}{ Number of Samples } \\
\hline Class Name & Training & Testing \\
\hline Road & 30 & 822 \\
\hline Tree & 30 & 1355 \\
\hline Grass & 30 & 821 \\
\hline Water & 30 & 379 \\
\hline Soil & 30 & 780 \\
\hline Total & 150 & 4157 \\
\hline
\end{tabular}

for testing. Fig. 4 shows pseudo-color image segments of the GU data. Table II shows the distribution of training and testing samples of this dataset.

\section{B. Experimental Setup}

1) Band-group and Multisource Fusions: The input hyperspectral data is normalised in the range of $[0,1]$. In order to generate DAP, the areas of 10,15 , and 20 and bounding box diagonals of 50,100, and 500 are used. A total of 13 profiles are generated for these area and diagonal parameters. In this work, $90 \%$ of the training samples are used to train the classifiers and the rest are validation samples to guide to a proper model in order to avoid over-training. In band-group fusion, we apply clustering on joint entropy and structural similarity metrics for band grouping. We compare performance of k-means and Expectation-Maximization (EM) clustering using Gaussian Mixture Models (GMM). Fig. 6(a) and (b) compare effectiveness of k-means and EM using GMM in classification by plotting of number of band groups vs overall accuracy (OA) for Houston and GU dataset respectively. In Fig. 6(a) EM using GMM performs better than k-means for Houston data and in Fig. 6(b) also EM using GMM clustering performs better than k-means for GU data. For both dataset EM using GMM clustering outperforms k-means, so we choose EM using GMM for band grouping. In our experiment, the initial $T$ seeds of the clustering algorithm are selected randomly. This selection process is performed ten times and the mean classification results are then reported.

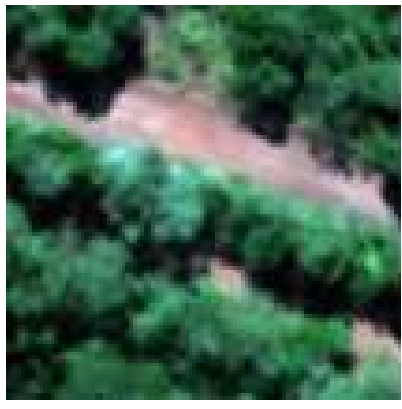

(a) $94 \times 98$

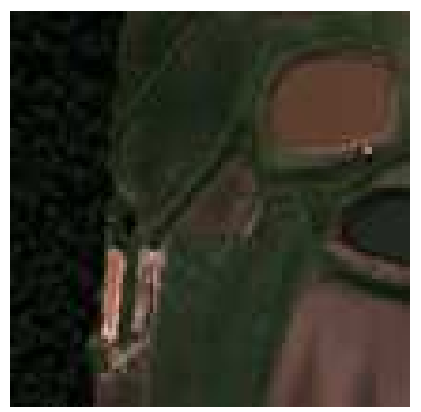

(c) $334 \times 479$

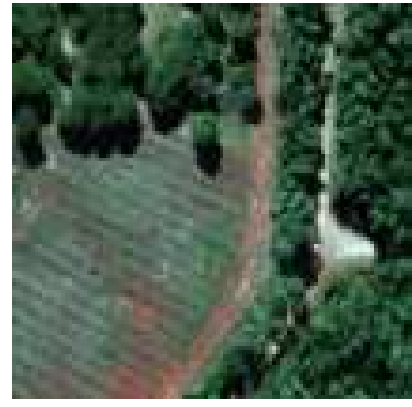

(b) $234 \times 272$

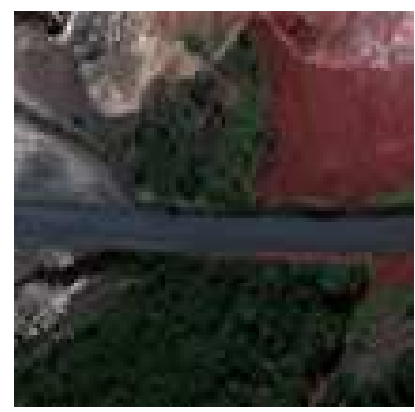

(d) $255 \times 368$

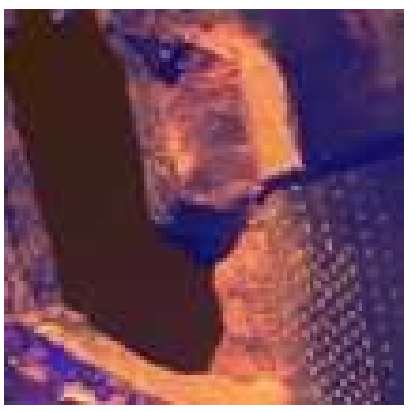

(e) $334 \times 392$

Fig. 4. Pseudo-color image of five areas arbitrarily selected from the GU HS image, showing samples of five classes, i.e., Road, Tree, Grass, Water and Soil. (a) Road, Tree and Grass; (b) Road, Tree, and Grass; (c) Tree, Grass, Water, and Soil; (d) Road, Tree, Grass, and Soil; (e) Water and Soil.

In multisource fusion, prior to applying the DAP to hyperspectral images, PCA is applied to the original hyperspectral data. In band-group fusion, PCA is applied to each group of bands separately. In both cases, the first few PCs are selected having at least $99 \%$ of their cumulative variance.

2) Generic Feature Extraction (GFeat): For NDVI we need to calculate near-infrared (NIR). In the case of the Houston data, we choose $797.90 \mathrm{~nm}$ (band 92) for NIR and $678.79 \mathrm{~nm}$ (band 30) for red. For the GU data, we choose $795.16 \mathrm{~nm}$ (band 42) for NIR and $679.46 \mathrm{~nm}$ (band 30) for red [24]. The description of two datasets are provided in Sections III-A1 and III-A2.

For the calculation of Entropy from HS we use a grayscale image. We generate the grayscale image from a pseudo-color RGB image. In the case of the Houston data, we use 693.10 $\mathrm{nm}$ (band 70) for red, $597.80 \mathrm{~nm}$ (band 50) for green, and $454.80 \mathrm{~nm}$ (band 20) for blue, respectively. For the GU data, we choose $650.84 \mathrm{~nm}$ (band 27) for red, $536.64 \mathrm{~nm}$ (band 15) for green, and $472.19 \mathrm{~nm}$ (band 8) for blue [24]. We choose 


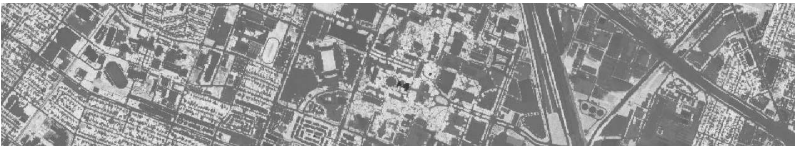

(a)

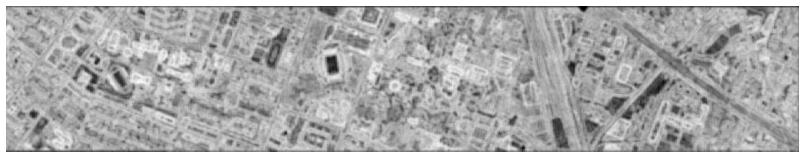

(b)

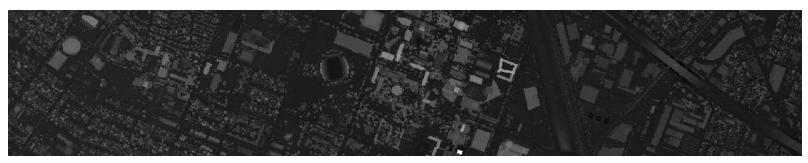

(c)

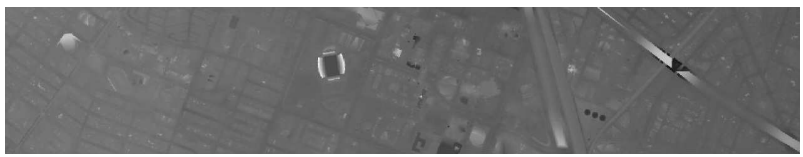

(d)

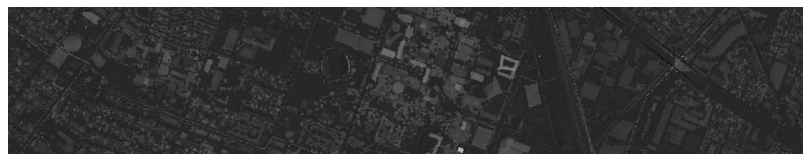

(e)

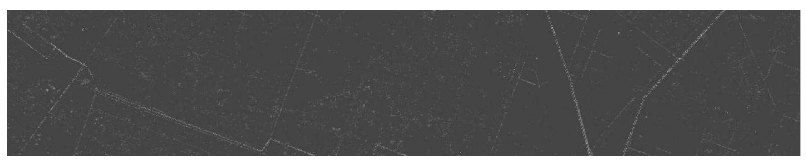

(f)

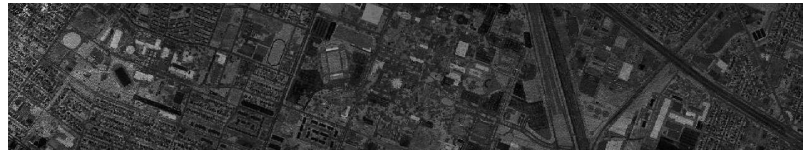

(g)

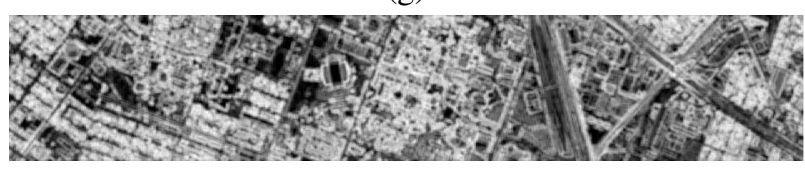

(h)

Fig. 5. Generic features from HS and LiDAR. (a) Normalised Difference Vegetation Index (NDVI), (b) Grayscale Entropy, (c) Digital Surface Model (DSM), (d) Digital Elevation Model (DEM), (e) Normalised Digital Surface Model (nDSM), (f) Difference between the first and last LiDAR return (PD), (g) Intensity, and (h) nDSM Entropy.

two different wavelength for red to compute NDVI and RGB image. According to [8], we prefer far-red rather than red to derive NDVI.

3) Classifiers: In classification, we use two classifiers e.g., Random Forest (RF) and Support Vector Machine (SVM).

For RF, the number of trees is set to 300 . The number of the prediction variable is set approximately to the square root of the number of input bands.

The SVM is trained with a Gaussian kernel by automatically setting the scale with Bayesian optimization model and penalty parameter (box constraint) in the range of $10^{-2}$ to $10^{4}$ during optimization. For Houston and GU datasets the best performing box constraints are 100 and 26.8 respectively. The classifier model is validated with 10 -fold cross validation.

The classification results are represented by overall accuracy (OA), average accuracy (AA) and the kappa coefficient [3]. The metrics OA and AA are in percentage. Kappa coefficient does not have a unit. The best OA, AA and Kappa results are shown in bold in Tables III, IV, V and VI . The number of features is put in parentheses.

The classification results are also presented by quantity disagreement, allocation disagreement and overall disagreement according to Pontius Jr et al. [28] in Tables III and V. The best overall disagreement results are shown in bold in Tables III and $\mathrm{V}$.

To represent the classification result of different methods, we use the following short-hand terms (i) DSM: Lidar DSM, (ii) HS: Hyperspectral data, (iii) DSM+HS: Stacked Hyperspectral and DSM, (iv) DAP(DSM): DAP applied to DSM, (v) DAP(HS): DAP applied to the principal components (having variance of $\geq 99 \%$ ) of HS, (vi) DAP(DSM)+DAP(HS): DAP(DSM) and DAP(HS) concatenated, (vii) DCA(HS) band-group fusion: band-group wise $\mathrm{DAP}(\mathrm{Spec})$ and $\mathrm{DAP}($ Spatial) fused and stacked, (viii) DCA(DSM,HS): DAP(DSM) and DAP(HS) fused, (x) GF: Generic features, (xi) DCA(HS)+DCA(DSM,HS)+GF: Stacked DCA(HS), DCA(DSM,HS) and GF.

\section{Results and Discussion on Houston Dataset}

1) RF Classification Results: Table III shows the classification accuracies obtained by different approaches using RF.

DSM represents elevation information of objects. So, the objects with different heights can easily be distinguished using this feature. For instance, "Tennis Court" has the highest classification accuracy because it has the lowest variations in DSM (e.g., standard deviation of 0.0013). On the contrary, "Parking Lot 1", "Parking Lot 2", and "Highway" have a very low accuracy in comparison to the average accuracy because their DSMs varies a lot within individual classes (standard deviation of $0.0630,0.0197$, and 0.0173 respectively). We use 144 raw bands of HS image. The accuracy using the raw bands is high because the objects of interest are made of different material compositions and show different reflectance properties. HS+DSM shows a higher OA than individual accuracy of DSM and HS. It confirms that they contain complementary information. Here, we get motivation of fusing them in an effective way other than just layer stacking.

DAP can significantly improve the classification accuracy because it can efficiently extract spatial structural information of different classes of interest which helps the classifier to differentiate them efficiently. For instance, DAP(DSM) significantly improves the classification accuracy (OA) of LiDAR by almost $32.84 \%$ which confirms the efficiency of DAP in feature extraction from raw LiDAR data. Unlike DAP(DSM), we apply PCA on HS to reduce number of planes because applying DAP on each plane of the HS is computationally very expensive and also all of the bands do not contain important information. Applying DAP on a band with almost no information will just lead to high computational cost as well as increase of redundant information. Since, we consider a few of 


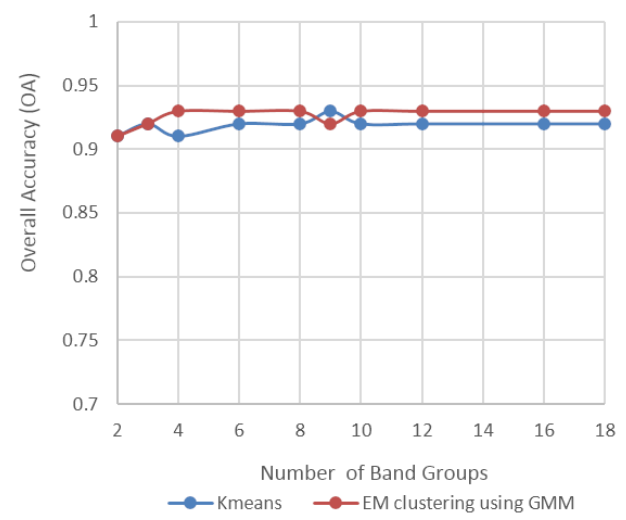

(a)

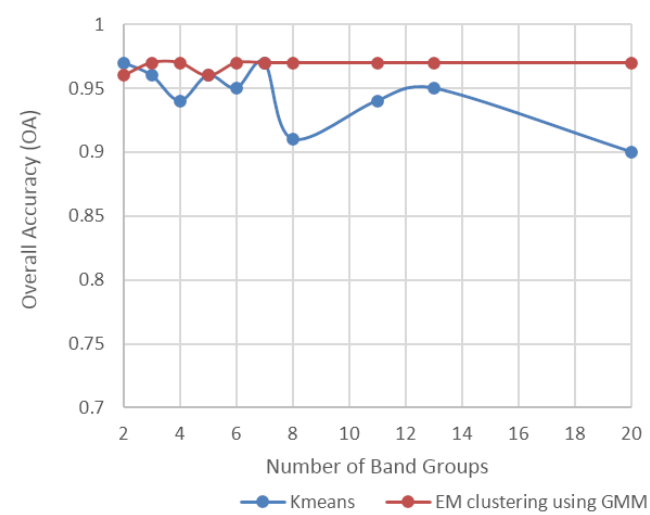

(b)

Fig. 6. Number of Band Groups vs Overall Accuracy of k-means and EM using GMM clustering. (a) Houston Data and (b) GU Data obtained by applying RF.

TABLE III

RF HOUSTON: CLASSIFICATION ACCURACIES BY DIFFERENT FEATURE AND FUSION OPTIONS. THE DIMENSION OF THE FINAL FEATURE VECTOR IS PROVIDED IN THE PARENTHESIS. IN THE CASE OF DCA(HS) THE NUMBER OF BAND GROUPS IS 9.

\begin{tabular}{|c|c|c|c|c|c|c|c|c|c|c|c|}
\hline & & $\begin{array}{l}\text { DSM } \\
\text { (1) }\end{array}$ & $\begin{array}{l}\text { HS } \\
(144)\end{array}$ & $\begin{array}{c}\text { DSM+ } \\
\text { HS } \\
(145)\end{array}$ & $\begin{array}{c}\text { DAP } \\
\text { (DSM) } \\
(13)\end{array}$ & $\begin{array}{l}\text { DAP } \\
\text { (HS) } \\
(39)\end{array}$ & $\begin{array}{c}\text { DAP(DSM) } \\
+ \text { DAP(HS) } \\
(52)\end{array}$ & $\begin{array}{c}\text { DCA(HS) } \\
\text { Band-group, } \\
(122)\end{array}$ & $\begin{array}{l}\text { DCA(DSM,HS) } \\
\text { Multisource } \\
\text { (18) }\end{array}$ & $\begin{array}{l}\text { GFeat } \\
(8)\end{array}$ & $\begin{array}{c}\text { Band-group+ } \\
\text { Multisource+ } \\
\text { GFeat } \\
(148)\end{array}$ \\
\hline 1 & Grass Healthy & 32.38 & 83.57 & 83.57 & 44.63 & 82.15 & 83.10 & 82.53 & 80.53 & 85.75 & 83.95 \\
\hline 2 & Grass Stressed & 17.48 & 98.40 & 98.31 & 54.51 & 91.92 & 94.08 & 99.34 & 97.27 & 98.21 & 99.62 \\
\hline 3 & Grass Synthetic & 55.25 & 98.02 & 98.22 & 94.26 & 100.00 & 100.00 & 100.00 & 100.00 & 99.41 & 100.00 \\
\hline 4 & Tree & 48.77 & 97.44 & 97.44 & 73.01 & 98.58 & 99.72 & 90.53 & 98.01 & 99.91 & 100.00 \\
\hline 5 & Soil & 35.98 & 96.40 & 96.88 & 79.64 & 98.77 & 99.53 & 99.34 & 99.43 & 88.35 & 99.81 \\
\hline 6 & Water & 53.15 & 97.20 & 97.20 & 81.82 & 99.30 & 99.30 & 95.10 & 97.20 & 86.71 & 95.80 \\
\hline 7 & Residential & 34.42 & 83.02 & 84.51 & 68.28 & 84.79 & 86.75 & 89.93 & 90.58 & 85.07 & 90.86 \\
\hline 8 & Commercial & 21.65 & 40.74 & 50.52 & 52.61 & 63.82 & 81.96 & 82.53 & 79.96 & 85.19 & 93.92 \\
\hline 9 & Road & 13.50 & 69.12 & 70.63 & 54.30 & 79.60 & 86.78 & 88.39 & 81.21 & 59.21 & 86.12 \\
\hline 10 & Highway & 14.38 & 57.43 & 58.30 & 31.85 & 77.70 & 59.85 & 70.75 & 57.53 & 46.91 & 78.57 \\
\hline 11 & Railway & 37.86 & 76.09 & 79.22 & 90.80 & 87.38 & 95.07 & 99.05 & 93.45 & 78.94 & 98.58 \\
\hline 12 & Parking Lot 1 & 11.34 & 49.28 & 58.89 & 44.19 & 72.14 & 65.42 & 90.68 & 77.23 & 65.42 & 91.35 \\
\hline 13 & Parking Lot 2 & 13.68 & 60.35 & 63.51 & 67.37 & 69.12 & 68.77 & 83.86 & 82.11 & 71.23 & 89.47 \\
\hline 14 & Tennis Court & 61.54 & 99.19 & 99.60 & 97.98 & 100.00 & 100.00 & 100.00 & 100.00 & 97.98 & 100.00 \\
\hline 15 & Running Track & 73.57 & 97.67 & 97.89 & 91.12 & 98.94 & 99.15 & 100.00 & 100.00 & 99.37 & 99.79 \\
\hline & OA & 30.52 & 77.46 & 79.86 & 63.36 & 85.16 & 86.53 & 90.36 & 87.10 & 81.27 & 93.05 \\
\hline & AA & 35.00 & 80.26 & 82.31 & 68.42 & 86.95 & 87.96 & 91.47 & 88.97 & 83.18 & 93.86 \\
\hline & Kappa & 0.25 & 0.76 & 0.78 & 0.60 & 0.84 & 0.85 & 0.90 & 0.86 & 0.80 & 0.92 \\
\hline & $\begin{array}{c}\text { Quantity } \\
\text { Disagreement }\end{array}$ & 0.03 & 0.05 & 0.05 & 0.06 & 0.05 & 0.06 & 0.06 & 0.06 & 0.06 & 0.05 \\
\hline & $\begin{array}{c}\text { Allocation } \\
\text { Disagreement }\end{array}$ & 0.64 & 0.16 & 0.14 & 0.29 & 0.10 & 0.08 & 0.05 & 0.07 & 0.12 & 0.02 \\
\hline & $\begin{array}{c}\text { Overall } \\
\text { Disagreement }\end{array}$ & 0.67 & 0.21 & 0.19 & 0.35 & 0.15 & 0.14 & 0.11 & 0.13 & 0.18 & 0.07 \\
\hline
\end{tabular}

the principal components in our experiment, DAP(HS) slightly reduces the classification accuracy but provides important structural information which is complementary to DAP(DSM) to some extent.

DCA(HS) represents the accuracy of band-group fusion. Fig. 6(a) shows how overall accuracy changes with the number of band groups in Table III. DCA(HS) fuses HS and DAP(HS) for every band group and shows higher accuracy in comparison to its individual inputs, e.g., HS and DAP(HS), as shown in Tables III and IV. This is because DCA removes correlation between classes within each input dataset, but increases correlation between sets. DCA also reduces noise as well as redundant information from the input data by considering significant singular values. If we apply band grouping based on joint entropy and structural similarity metrics, it significantly improves the accuracy (OA) from raw HS band by more than $12.90 \%$ with dimensionality reduction. In this case we achieve the highest accuracy for 8 band groups.

DCA(DSM, HS) which we refer as multisource fusion slightly reduces accuracy than band-group fusion because it fuses DAP(DSM) and DAP(HS), i.e., both of them from spatial domain, so they have less mutual information than fusing spectral and spatial domains. On the contrary, the fused data has complementary information with band-group fusion output and GFeat. The stacked form of the outputs of band-group fusion and multisource fusion units and GF are concatenated to get final feature of each sample. The final feature achieves classification accuracy of $93.05 \%$ because the components 
TABLE IV

SVM HOUSTON: CLASSIFICATION ACCURACIES BY DIFFERENT FEATURE AND FUSION OPTIONS. THE DIMENSION OF THE FINAL FEATURE VECTOR IS PROVIDED IN THE PARENTHESIS. IN THE CASE OF DCA(HS) THE NUMBER OF BAND GROUPS IS 9.

\begin{tabular}{|c|c|c|c|c|c|c|c|c|c|c|c|}
\hline & & $\begin{array}{c}\text { DSM } \\
(1)\end{array}$ & $\begin{array}{c}\text { HS } \\
(144)\end{array}$ & $\begin{array}{c}\text { DSM+ } \\
\text { HS } \\
(145)\end{array}$ & $\begin{array}{c}\text { DAP } \\
(\text { DSM) } \\
(13)\end{array}$ & $\begin{array}{c}\text { DAP } \\
(\text { HS) } \\
(39)\end{array}$ & $\begin{array}{c}\text { DAP(DSM) } \\
+ \text { DAP(HS) } \\
(52)\end{array}$ & $\begin{array}{c}\text { DCA(HS) } \\
\text { Band-group, } \\
(122)\end{array}$ & $\begin{array}{c}\text { DCA } \\
\text { (DSM,HS) } \\
\text { Multisource, } \\
(18)\end{array}$ & $\begin{array}{c}\text { GFeat } \\
(\text { 08) }\end{array}$ & $\begin{array}{c}\text { Band-group+ } \\
\text { Multisource+ } \\
\text { GFeat } \\
(148)\end{array}$ \\
\hline 1 & Grass Healthy & 30.58 & 83.10 & 84.14 & 44.25 & 81.77 & 81.91 & 81.01 & 81.29 & 86.32 & 82.05 \\
\hline 2 & Grass Stressed & 18.61 & 98.21 & 98.31 & 35.53 & 97.56 & 96.80 & 99.44 & 98.40 & 95.86 & 99.53 \\
\hline 3 & Grass Synthetic & 54.85 & 99.41 & 99.41 & 94.26 & 100.00 & 100.00 & 100.00 & 100.00 & 97.62 & 100.00 \\
\hline 4 & Tree & 48.58 & 98.20 & 98.77 & 63.73 & 95.45 & 99.81 & 90.15 & 94.51 & 97.06 & 98.96 \\
\hline 5 & Soil & 40.44 & 94.98 & 94.98 & 71.69 & 97.06 & 99.24 & 99.15 & 95.36 & 59.75 & 98.96 \\
\hline 6 & Water & 50.35 & 88.81 & 90.91 & 74.13 & 96.50 & 99.30 & 90.91 & 91.61 & 74.13 & 99.30 \\
\hline 7 & Residential & 36.75 & 88.53 & 88.43 & 56.62 & 90.21 & 91.14 & 87.50 & 94.87 & 81.62 & 91.88 \\
\hline 8 & Commercial & 23.84 & 42.64 & 69.14 & 60.11 & 51.28 & 66.67 & 50.43 & 63.34 & 71.32 & 79.11 \\
\hline 9 & Road & 12.28 & 56.00 & 72.62 & 44.76 & 77.90 & 85.27 & 80.55 & 74.13 & 44.00 & 82.53 \\
\hline 10 & Highway & 16.51 & 62.64 & 67.76 & 27.03 & 65.93 & 67.57 & 83.20 & 69.59 & 31.85 & 84.56 \\
\hline 11 & Railway & 37.00 & 79.22 & 87.67 & 86.05 & 88.33 & 98.20 & 95.16 & 91.94 & 68.79 & 96.87 \\
\hline 12 & Parking Lot 1 & 8.74 & 54.47 & 46.40 & 44.48 & 76.75 & 82.32 & 73.58 & 72.91 & 39.00 & 95.00 \\
\hline 13 & Parking Lot 2 & 14.39 & 42.81 & 44.21 & 72.63 & 65.96 & 72.98 & 76.84 & 71.93 & 74.39 & 83.16 \\
\hline 14 & Tennis Court & 86.64 & 98.79 & 98.38 & 98.79 & 100.00 & 100.00 & 99.60 & 99.60 & 80.16 & 100.00 \\
\hline 15 & Running Track & 74.21 & 95.98 & 97.25 & 95.77 & 100.00 & 100.00 & 97.89 & 100.00 & 92.39 & 98.73 \\
\hline & OA & 31.50 & 77.49 & 81.93 & 58.41 & 83.86 & 88.17 & 85.48 & 85.15 & 70.38 & $\mathbf{9 1 . 7 4}$ \\
\hline & AA & 36.92 & 78.92 & 82.56 & 64.65 & 85.65 & 89.48 & 87.03 & 86.63 & 72.95 & $\mathbf{9 2 . 7 1}$ \\
\hline & Kappa & 0.26 & 0.76 & 0.80 & 0.55 & 0.82 & 0.87 & 0.84 & 0.84 & 0.68 & $\mathbf{0 . 9 1}$ \\
\hline
\end{tabular}

TABLE V

RF GU: CLASSIFICATION ACCURACIES BY DIFFERENT FEATURE AND FUSION OPTIONS. THE DIMENSION OF THE FINAL FEATURE VECTOR IS PROVIDED IN THE PARENTHESIS. IN THE CASE OF DCA(HS) THE NUMBER OF BAND GROUPS IS 7.

\begin{tabular}{|c|c|c|c|c|c|c|c|c|c|c|c|}
\hline & & $\begin{array}{c}\text { DSM } \\
(1)\end{array}$ & $\begin{array}{c}\text { HS } \\
(62)\end{array}$ & $\begin{array}{c}\text { DSM+ } \\
\text { HS } \\
(63)\end{array}$ & $\begin{array}{c}\text { DAP } \\
(\text { DSM) } \\
(13)\end{array}$ & $\begin{array}{c}\text { DAP } \\
(\text { HS) } \\
(39)\end{array}$ & $\begin{array}{c}\text { DAP(DSM) } \\
\text { +DAP(HS) } \\
(52)\end{array}$ & $\begin{array}{c}\text { DCA(HS) } \\
\text { Band-group, } \\
(52)\end{array}$ & $\begin{array}{c}\text { DCA } \\
\text { (DSM,HS) } \\
\text { Multisource } \\
(8)\end{array}$ & $\begin{array}{c}\text { GFeat } \\
(8)\end{array}$ & $\begin{array}{c}\text { Band-group+ } \\
\text { Multisource+ } \\
\text { GFeat } \\
(68)\end{array}$ \\
\hline 1 & Road & 51.78 & 92.52 & 92.39 & 78.04 & 89.69 & 92.88 & 98.39 & 90.92 & 95.83 & 98.40 \\
\hline 2 & Tree & 42.39 & 83.91 & 86.14 & 81.54 & 70.11 & 84.70 & 87.41 & 85.99 & 91.95 & 95.26 \\
\hline 3 & Grass & 58.91 & 88.12 & 90.27 & 71.68 & 80.66 & 91.53 & 95.13 & 87.61 & 95.95 & 93.30 \\
\hline 4 & Water & 95.25 & 100.00 & 100.00 & 92.08 & 100.00 & 100.00 & 100.00 & 100.00 & 100.00 & 100.00 \\
\hline 5 & Soil & 69.23 & 94.36 & 94.62 & 85.38 & 76.54 & 90.64 & 99.74 & 88.85 & 98.33 & 100.00 \\
\hline & OA & 57.23 & 89.82 & 91.00 & 80.66 & 79.89 & 90.11 & 94.47 & 89.08 & 95.41 & $\mathbf{9 6 . 8 2}$ \\
\hline & AA & 63.51 & 91.78 & 92.68 & 81.74 & 83.40 & 91.95 & 96.13 & 90.67 & 96.41 & $\mathbf{9 7 . 3 9}$ \\
\hline & Kappa & 0.46 & 0.87 & 0.88 & 0.75 & 0.74 & 0.87 & 0.93 & 0.86 & 0.94 & $\mathbf{0 . 9 6}$ \\
\hline & $\begin{array}{l}\text { Quantity } \\
\text { Disagreement }\end{array}$ & 0.05 & 0.04 & 0.03 & 0.05 & 0.07 & 0.05 & 0.02 & 0.05 & 0.02 & 0.02 \\
\hline & $\begin{array}{l}\text { Allocation } \\
\text { Disagreement }\end{array}$ & 0.37 & 0.07 & 0.06 & 0.12 & 0.12 & 0.05 & 0.03 & 0.05 & 0.02 & 0.02 \\
\hline & $\begin{array}{l}\text { Overall } \\
\text { Disagreement }\end{array}$ & 0.42 & 0.11 & 0.09 & 0.17 & 0.19 & 0.10 & 0.05 & 0.10 & $\mathbf{0 . 0 4}$ & $\mathbf{0 . 0 4}$ \\
\hline
\end{tabular}

in this signature contains complementary information. Grass Stressed, Grass Synthetic, Tree, Soil, Water, Tennis Court, Running Track have more than $95 \%$ accuracy while Grass Healthy (OA: 83.95\%), Residential (OA: 90.86\%) and Parking Lot 2 (OA: 89.47\%) have comparatively low accuracy.

Our DCA(HS) improves the classification accuracies of "Grass healthy", "Commercial", "Road", "Parking lot1" and "Parking lot2" compared to individual HS or DAP(HS). Also DCA(DSM,HS) improves the classification accuracies for above mentioned classes than using only DAP(DSM). The reason is that DCA(DSM,HS) fuses spatial information of both HS and DSM in an effective manner. According to [14] for the class "highway" elevation value changes along the whole sample set. The combination of DCA(HS), DCA(DSM,HS) and GFeat significantly improves the accuracies of "highway" because they contain complementary information for that class.
2) SVM Classification Results: Table IV shows the classification results obtained by the SVM classifier, which performs well as the number of features increases. SVM outperforms RF when we used both HS raw data and DSM, or the combination of attribute profile extracted from HS image and DSM. On the contrary, RF outperforms SVM in band-group fusion, multisource fusion and generic feature units. In consideration of overall system performance, RF outperforms SVM which is also confirmed by other works in the literature [16].

\section{Results and Discussion on GU Dataset}

1) RF Classification Results: Table V shows the classification results obtained by the RF classifier on the GU dataset. The overall accuracy on GU data is $96.82 \%$. This is significantly higher than the accuracy on Houston data because the number of classes are only one-third of the number of Houston data. As a result, the confusion between classes is lower on the 
TABLE VI

SVM GU: CLASSIFICATION ACCURACIES BY DIFFERENT FEATURE AND FUSION OPTIONS. THE DIMENSION OF THE FINAL FEATURE VECTOR IS PROVIDED IN THE PARENTHESIS. IN THE CASE OF DCA(HS) THE NUMBER OF BAND GROUPS IS 7.

\begin{tabular}{|c|c|c|c|c|c|c|c|c|c|c|c|}
\hline & & $\begin{array}{c}\text { DSM } \\
(1)\end{array}$ & $\begin{array}{c}\text { HS } \\
(62)\end{array}$ & $\begin{array}{c}\text { DSM+ } \\
\text { HS } \\
(63)\end{array}$ & $\begin{array}{c}\text { DAP } \\
\text { (DSM) } \\
(13)\end{array}$ & $\begin{array}{c}\text { DAP } \\
\text { (HS) } \\
(39)\end{array}$ & $\begin{array}{c}\text { DAP(DSM) } \\
\text { +DAP(HS) } \\
(52)\end{array}$ & $\begin{array}{c}\text { DCA(HS) } \\
\text { Band-group, } \\
(52)\end{array}$ & $\begin{array}{c}\text { DCA } \\
\text { (DSM,HS) } \\
\text { Multisource } \\
(8)\end{array}$ & $\begin{array}{c}\text { GFeat } \\
(8)\end{array}$ & $\begin{array}{c}\text { Band-group+ } \\
\text { Multisource+ } \\
\text { GFeat } \\
(68)\end{array}$ \\
\hline 1 & Road & 46.50 & 94.11 & 94.60 & 80.98 & 92.27 & 96.32 & 95.47 & 96.44 & 91.53 & 98.74 \\
\hline 2 & Tree & 46.41 & 89.94 & 94.25 & 85.06 & 75.22 & 90.37 & 89.87 & 92.17 & 94.40 & 96.57 \\
\hline 3 & Grass & 64.48 & 89.76 & 89.13 & 82.17 & 78.00 & 84.45 & 86.77 & 84.07 & 96.59 & 94.94 \\
\hline 4 & Water & 82.06 & 100.00 & 100.00 & 80.47 & 100.00 & 100.00 & 100.00 & 100.00 & 100.00 & 100.00 \\
\hline 5 & Soil & 76.03 & 97.05 & 97.44 & 87.82 & 78.21 & 94.87 & 96.71 & 90.90 & 95.00 & 95.92 \\
\hline & OA & 58.67 & 92.00 & 94.47 & 83.81 & 81.91 & 92.13 & 92.56 & 91.94 & 94.88 & $\mathbf{9 6 . 8 7}$ \\
\hline & AA & 63.09 & 94.17 & 95.08 & 83.30 & 84.74 & 93.20 & 93.76 & 92.72 & 95.50 & $\mathbf{9 7 . 2 4}$ \\
\hline & Kappa & 0.47 & 0.91 & 0.93 & 0.79 & 0.77 & 0.90 & 0.90 & 0.90 & 0.93 & $\mathbf{0 . 9 6}$ \\
\hline
\end{tabular}

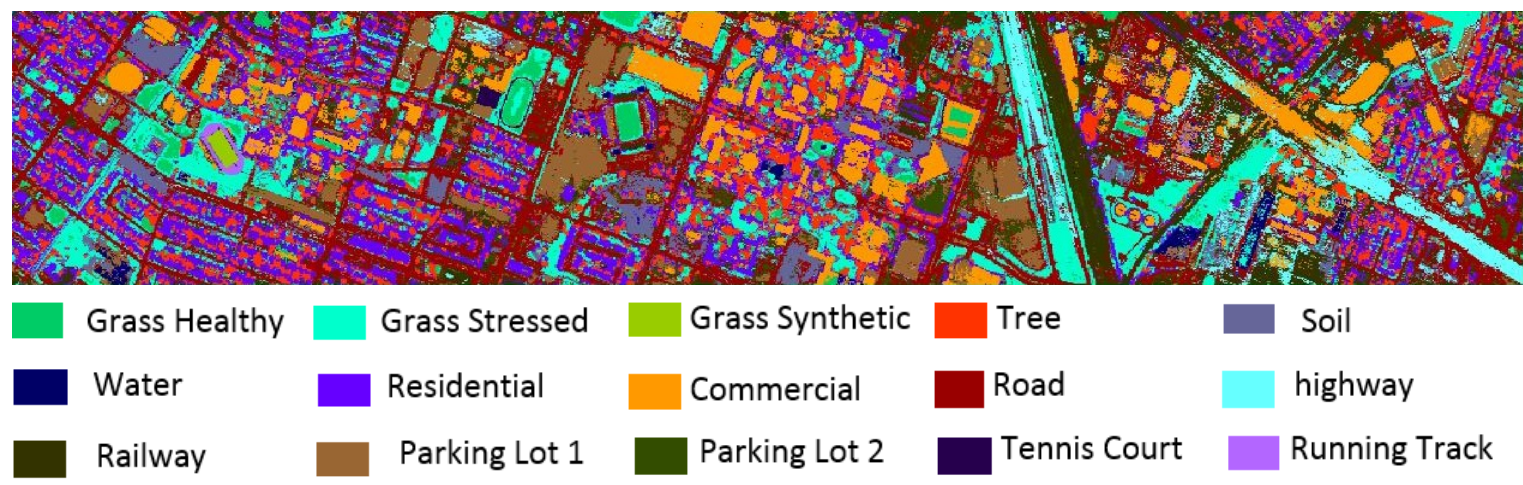

(a)
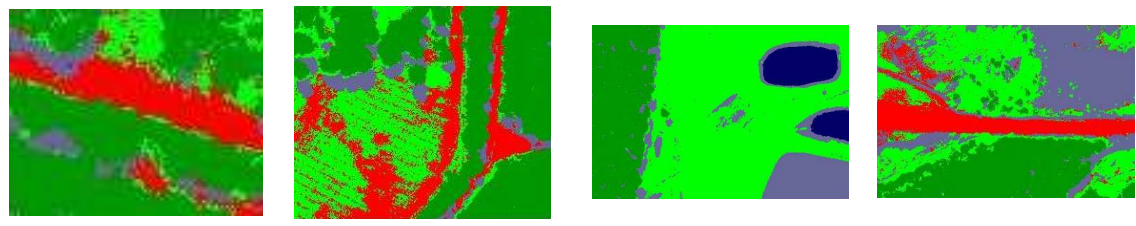

Tree

Road

Grass

Water

Soil

(b)

Fig. 7. Classification maps generated by the output of the proposed method using RF (a) Houston data. (b) GU data

GU data. If we look at the class-wise accuracy, for the feature DSM, "Tree" has the lowest accuracy because it lacks a solid structure. "Grass" and "Road" lead to lower classification for DAP (DSM) for the reason that their height are almost similar (close to the ground), so only using the height feature confuses between these two classes. Our DCA(HS) band-group fusion method achieves higher accuracies on every class than using the raw HS bands. Fig. 6(b) shows how overall accuracy changes with the number of band groups used in DCA(HS) on GU Data. The highest overall accuracy is obtained for GU Data when there are 7 groups. Combining DCA(HS) bandgroup fusion, DCA(DSM,HS) multisource fusion and GFeat improves the accuracies of all classes as evident from $\mathrm{V}$.

2) SVM Classification Results: Table VI shows the classification results obtained by the SVM classifier. Classification of raw data from DSM and HS, attribute profile, and fusion output shows that SVM outperforms RF. In this dataset, "Water" is classified accurately for HS and HS-derived features because it is not confused with other classes due to its formation material. On the contrary, "Grass" is mostly confused with "Road" and "Tree". The reason behind this is that "Grass" and "Road" are similar in elevation and "Grass" and "Tree" are similar in texture.

To evaluate the contribution of band-group fusion towards the feature extraction from HS data, we report the results in Table III to Table VI with and without this step. It is clearly seen that band-group fusion unit improves the quality of HS-derived feature as well as classification accuracy. RF Houston, SVM Houston, RF GU and SVM GU improve accuracy respectively by $12.90 \%, 7.99 \%, 4.65 \%$ and $0.56 \%$ in comparison with raw HS data. For GU, the improvement is not as significant as Houston because it already has very high accuracies $(89.82 \%, 92 \%)$.

Fusion of DSM-derived and HS-derived features in multisource fusion also improves the accuracy of their individual features and reduces the dimensionality of features. They also have a significant role on the overall performance. Multisource fusion in RF Houston data improves $0.57 \%$ accuracy from 
$\mathrm{DAP}(\mathrm{DSM})+\mathrm{DAP}(\mathrm{HS})$ while the feature dimensionality is reduced from 52 to 18 .

Finally, we investigate the contribution from generic features. These features have complementary information with band-group fusion and multisource derived feature. Adding these features leads to $93.05 \%, 91.74 \%, 96.82 \%$ and $96.87 \%$ accuracy on RF Houston, SVM Houston, RF GU and SVM GU respectively. Fig. 7 shows classification maps of the proposed method for Houston and GU data.

\section{E. Performance Comparison}

Finally, we compare our method with five state-of-the-art approaches. Most of these approaches used Houston Data. To be fully comparable with other approaches, we kept the standard settings of the training and testing samples.

Man et al. [3] fused texture based grey-level co-occurrence matrix (GLCM) and NDVI from HS with LiDAR nDSM and intensity in pixel level and classified using SVM. As our method only uses pixel-based fusion and classification, the classification accuracies of pixel-based fusion obtained by SVM in [3] are included in Table VII. Khodadadzadeh et al. [13] used attribute profile on LiDAR DSM and extended attribute profile on PCs extracted from HS data and integrated them by a subspace multinomial logistic regression in a flexible manner without using any regularization parameters. In [14], Extinction Profile (EP) based spatial features are extracted from both HS and LiDAR data, then fused by layer stacking/graph based fusion method before fed to CNN. In [15] a two branch CNN was employed for the fusion of HS and LiDAR data where a two-tunnel CNN derived spatial and spectral features from HS and a cascade block CNN were designed for feature extraction of LiDAR data.

Our band-group fusion unit successfully extracted a discriminative signature from HS with the reduction of dimensionality, by achieving $90.36 \%$ accuracy using RF. This is significantly higher than the existing methods. For instance, [13] obtained $84.40 \%$ accuracy using spectral and spatial feature from HS. Using EP on HS data, [14] and [16] obtained $80.36 \%$ accuracy while feature vector dimensionality is 213 . Using the twotunnel CNN framework for the spectral and spatial feature extraction from HS, [15] obtained $84.08 \%$ accuracy. The reason behind this is that using band grouping our band-group fusion method extracts useful local spectral and spatial features. These features are effectively fused by DCA which optimises both between-class variation and within-class similarity with the reduction of dimensionality.

TABLE VII

COMPARISON WITH OTHER STUDIES IN THE LITERATURE.

\begin{tabular}{|c|c|c|c|c|c|c|}
\hline & $\begin{array}{c}\text { Man } \\
\text { et al. } \\
{[3]}\end{array}$ & $\begin{array}{c}\text { Khodadad } \\
\text {-zadeh } \\
\text { et al. } \\
{[13]}\end{array}$ & $\begin{array}{l}\text { Ghamisi } \\
\text { et al. } \\
{[14]}\end{array}$ & $\begin{array}{l}\text { Rasti } \\
\text { et al. } \\
{[16]}\end{array}$ & $\begin{array}{l}\text { Xu } \\
\text { et al. } \\
{[15]}\end{array}$ & $\begin{array}{l}\text { Proposed } \\
\text { Method }\end{array}$ \\
\hline OA & 87.60 & 90.65 & 91.02 & 91.30 & 87.98 & $\mathbf{9 3 . 0 5}$ \\
\hline AA & - & 91.11 & 91.82 & 91.95 & 90.11 & $\mathbf{9 3 . 8 6}$ \\
\hline Kappa & 0.87 & 0.90 & 0.90 & 0.91 & 0.91 & $\mathbf{0 . 9 2}$ \\
\hline
\end{tabular}

\section{CONCLUSION}

In this paper, we propose a novel method for the classification of complex land cover. This method includes novel feature extraction and fusion techniques. This study contributes in the field of HS and LiDAR fusion in the following ways: it devises a novel band grouping technique, fusion of spectral and spatial features from HS data, and fusion of LiDAR-derived DSM and HS data. The extended fusion technique increases inter-class variance and decreases intra-class variance. Band grouping proves that it strengthens the features produced from the HS data. We also adopt several discriminative features which improve classification accuracy significantly. A combined signature generated by band-group fusion, multisource fusion and generic features provides a competent accuracy compared to other state-of-the-art methods. Validated on both Houston benchmark dataset and a GU dataset, our approach outperforms the existing methods with significant margins. Therefore, the proposed model is a new and effective approach for remote sensing HS and LiDAR data fusion.

\section{ACKNOWLEDGMENT}

The authors would like to express their appreciation to the National Center for Airborne Laser Mapping for providing the Houston dataset, Prof. Naoto Yokoya from Technical University of Munich (TUM) for providing shadow-removed hyperspectral Houston data, and Prof. Jorg Hacker from Flinders University for collecting the Airborne HS and LiDAR Point Cloud from which we prepared GU dataset. Furthermore, the authors greatly appreciate Dr. Jun Li from Sun Yat-Sen University for sharing the EMAP code with us and Pedram Ghamisi from Technical University of Munich (TUM) for technical support.

\section{REFERENCES}

[1] M. Dalponte, H. O. Ørka, L. T. Ene, T. Gobakken, and E. Næsset, "Tree crown delineation and tree species classification in boreal forests using hyperspectral and ALS data," Remote Sensing of Environment, vol. 140, no. Supplement C, pp. $306-317,2014$.

[2] A. Ferraz, S. Saatchi, C. Mallet, and V. Meyer, "LiDAR detection of individual tree size in tropical forests," $R e$ mote Sensing of Environment, vol. 183, no. Supplement C, pp. 318 - 333, 2016.

[3] Q. Man, P. Dong, and H. Guo, "Pixel- and featurelevel fusion of hyperspectral and LiDAR data for urban land-use classification," International Journal of Remote Sensing, vol. 36, no. 6, pp. 1618-1644, 2015.

[4] S. Luo, C. Wang, X. Xi, H. Zeng, D. Li, S. Xia, and P. Wang, "Fusion of airborne discrete-return LiDAR and hyperspectral data for land cover classification," Remote Sensing, vol. 8, no. 1, p. 3, 2016.

[5] C. Debes, A. Merentitis, R. Heremans, J. Hahn, N. Frangiadakis, T. van Kasteren, W. Liao, R. Bellens, A. Pizurica, S. Gautama, W. Philips, S. Prasad, Q. Du, and F. Pacifici, "Hyperspectral and lidar data fusion: Outcome of the 2013 grss data fusion contest," IEEE 
Journal of Selected Topics in Applied Earth Observations and Remote Sensing, vol. 7, no. 6, pp. 2405-2418, 2014.

[6] I. Klein, U. Gessner, and C. Kuenzer, "Regional land cover mapping and change detection in central asia using MODIS time-series," Applied Geography, vol. 35, no. 1-2, pp. $219-234,2012$.

[7] E. Adam, O. Mutanga, J. Odindi, and E. M. AbdelRahman, "Land-use/cover classification in a heterogeneous coastal landscape using rapideye imagery: evaluating the performance of random forest and support vector machines classifiers," International Journal of Remote Sensing, vol. 35, no. 10, pp. 3440-3458, 2014.

[8] M. Dalponte, L. Bruzzone, and D. Gianelle, "Tree species classification in the southern alps based on the fusion of very high geometrical resolution multispectral/hyperspectral images and LiDAR data," Remote Sensing of Environment, vol. 123, no. Supplement C, pp. $258-270,2012$.

[9] S. Morchhale, V. P. Pauca, R. J. Plemmons, and T. C. Torgersen, "Classification of pixel-level fused hyperspectral and LiDAR data using deep convolutional neural networks," in 8th Workshop on Hyperspectral Image and Signal Processing: Evolution in Remote Sensing (WHISPERS), Aug 2016, pp. 1-5.

[10] Q. Man, P. Dong, H. Guo, G. Liu, and R. Shi, "Light detection and ranging and hyperspectral data for estimation of forest biomass: a review," Journal of Applied Remote Sensing, vol. 8, no. 1, p. 081598, 2014.

[11] P. Ghamisi, J. A. Benediktsson, and S. Phinn, "Landcover classification using both hyperspectral and LiDAR data," International Journal of Image and Data Fusion, vol. 6, no. 3, pp. 189-215, 2015.

[12] B. Bigdeli, F. Samadzadegan, and P. Reinartz, "Fusion of hyperspectral and LiDAR data using decision templatebased fuzzy multiple classifier system," International Journal of Applied Earth Observation and Geoinformation, vol. 38, pp. 309-320, 2015.

[13] M. Khodadadzadeh, J. Li, S. Prasad, and A. Plaza, "Fusion of hyperspectral and LiDAR remote sensing data using multiple feature learning," IEEE Journal of Selected Topics in Applied Earth Observations and Remote Sensing, vol. 8, no. 6, pp. 2971-2983, 2015.

[14] P. Ghamisi, B. Höfle, and X. X. Zhu, "Hyperspectral and LiDAR data fusion using extinction profiles and deep convolutional neural network," IEEE Journal of Selected Topics in Applied Earth Observations and Remote Sensing, vol. 10, no. 6, pp. 3011-3024, June 2017.

[15] X. Xu, W. Li, Q. Ran, Q. Du, L. Gao, and B. Zhang, "Multisource remote sensing data classification based on convolutional neural network," IEEE Transactions on Geoscience and Remote Sensing, vol. 56, no. 2, pp. $937-$ 949, Feb 2018.

[16] B. Rasti, P. Ghamisi, J. Plaza, and A. Plaza, "Fusion of hyperspectral and LiDAR data using sparse and low-rank component analysis," IEEE Transactions on Geoscience and Remote Sensing, vol. 55, no. 11, pp. 6354-6365, Nov 2017.

[17] W. Liao, A. Pižurica, R. Bellens, S. Gautama, and
W. Philips, "Generalized graph-based fusion of hyperspectral and lidar data using morphological features," IEEE Geoscience and Remote Sensing Letters, vol. 12, no. 3, pp. 552-556, March 2015.

[18] M. Haghighat, M. Abdel-Mottaleb, and W. Alhalabi, "Discriminant correlation analysis: Real-time feature level fusion for multimodal biometric recognition," IEEE Transactions on Information Forensics and Security, vol. 11, no. 9, pp. 1984-1996, Sept 2016.

[19] T. K. Kim, J. Kittler, and R. Cipolla, "Discriminative learning and recognition of image set classes using canonical correlations," IEEE Transactions on Pattern Analysis and Machine Intelligence, vol. 29, no. 6, pp. 1005-1018, June 2007.

[20] M. D. Mura, J. A. Benediktsson, B. Waske, and L. Bruzzone, "Morphological attribute profiles for the analysis of very high resolution images," IEEE Transactions on Geoscience and Remote Sensing, vol. 48, no. 10, pp. 3747-3762, Oct 2010.

[21] Z. Wang, A. C. Bovik, H. R. Sheikh, and E. P. Simoncelli, "Image quality assessment: from error visibility to structural similarity," IEEE Transactions on Image Processing, vol. 13, no. 4, pp. 600-612, April 2004.

[22] I. Jolliffe, Principal component analysis. Wiley Online Library, 2002.

[23] M. Haghighat, M. Abdel-Mottaleb, and W. Alhalabi, "Discriminant correlation analysis for feature level fusion with application to multimodal biometrics," March 2016, pp. $1866-1870$.

[24] F. Jahan and M. Awrangjeb, "Pixel-based land cover classification by fusing hyperspectral and lidar data," ISPRS - International Archives of the Photogrammetry, Remote Sensing and Spatial Information Sciences, vol. XLII-2/W7, pp. 711-718, 2017.

[25] M. A. Holm AM, Burnside DG, "The development of a system for monitoring trend in range condition in the arid shrublands of western australia," The Australian Rangeland Journal, vol. 9, pp. $14-20,1987$.

[26] R. C. Gonzalez, R. E. Woods, and S. L. Eddins, Digital Image Processing Using MATLAB. Upper Saddle River, NJ, USA: Prentice-Hall, Inc., 2003.

[27] D. F. Contest. 2013 IEEE GRSS Data Fusion Contest Fusion of Hyperspectral and LiDAR Data. [Online]. Available: http://hyperspectral.ee.uh.edu/

[28] R. G. P. Jr and M. Millones, "Death to kappa: birth of quantity disagreement and allocation disagreement for accuracy assessment," International Journal of Remote Sensing, vol. 32, no. 15, pp. 4407-4429, 2011. [Online]. Available: https://doi.org/10.1080/01431161.2011.552923 


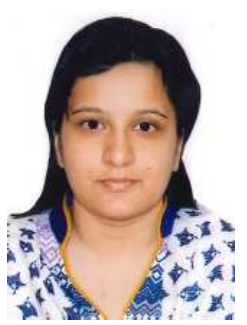

Farah Jahan Farah Jahan received the B.Sc.(Hons.) degree in Computer Science \& Engineering from University of Chittagong, Chittagong, Bangladesh in 2008. She received the Master of Engineering degree in Information \& Telecommunication Engineering from Korea Aerospace University, South Korea in 2011. She is currently pursuing Ph.D. study in Griffith University, Australia. She is an Assistant Professor in the department of Computer Science \& Engineering in University of Chittagong, Chittagong, Bangladesh. Her research interest includes pattern recognition, machine learning, computer vision and data fusion in the field of remote sensing.

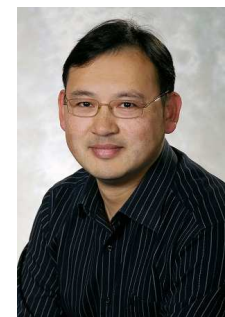

Jun Zhou received the B.S. degree in computer science and the B.E. degree in international business from Nanjing University of Science and Technology, Nanjing, China, in 1996 and 1998, respectively. He received the M.S. degree in computer science from Concordia University, Montreal, Canada, in 2002, and the Ph.D. degree from the University of Alberta, Edmonton, Canada, in 2006. He is a senior lecturer in the School of Information and Communication Technology at Griffith University, Nathan, Australia. Previously, he had been a research fellow in the Research School of Computer Science at the Australian National University, Canberra, Australia, and a researcher in the Canberra Research Laboratory, NICTA, Australia. His research interests include pattern recognition, computer vision and spectral imaging with their applications to remote sensing and environmental informatics.

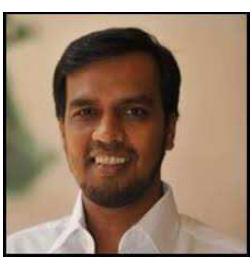

Mohammad Awrangjeb received his $\mathrm{PhD}$ degree from Monash University. Before joining Griffith University as a Lecturer, Dr. Awrangjeb worked as a (Senior) Research Fellow at Federation University Australia, Monash University and University of Melbourne. He is a recipient of the Discovery Early Career Researcher Award by the Australian Research Council for the period 2012-15. His research interest includes automatic feature extraction and matching, multimedia security and image processing, automatic building extraction and 3D modelling from

remote sensing data.

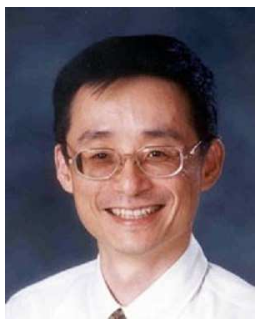

Yongsheng Gao received the B.Sc. and M.Sc. degrees in electronic engineering from Zhejiang University, China, in 1985 and 1988, respectively, and the Ph.D. degree in computer engineering from Nanyang Technological University, Singapore. He is currently a Professor with the School of Engineering at Griffith University, Australia, and Director of Australian Research Council Research Hub for Driving Farming Productivity and Disease Prevention. He had been the Leader of Biosecurity Group, Queensland Research Laboratory, National ICT Australia (ARC Centre of Excellence), a consultant of Panasonic Singapore Laboratories, and an Assistant Professor in the School of Computer Engineering, Nanyang Technological University, Singapore. His research interests include smart farming, intelligent agriculture, biosecurity, face recognition, biometrics, image retrieval, computer vision, pattern recognition, environmental informatics, and medical imaging. He is a senior member of IEEE. 